\title{
Dust observations of Comet 9P/Tempel 1 at the time of the Deep Impact ${ }^{\star}$
}

\author{
G. P. Tozzi ${ }^{1}$, H. Boehnhardt ${ }^{2}$, L. Kolokolova ${ }^{3}$, T. Bonev ${ }^{4}$, E. Pompei ${ }^{5}$, S. Bagnulo $^{6}$, N. Ageorges ${ }^{5}$, L. Barrera ${ }^{7}$,

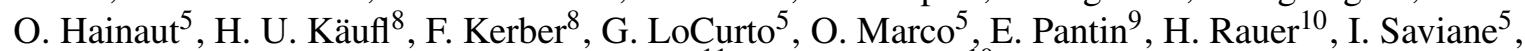 \\ C. Sterken ${ }^{11}$, and M. Weiler ${ }^{10}$ \\ 1 INAF - Osservatorio Astrofisico di Arcetri, Largo E. Fermi 5, 50125 Firenze, Italy \\ e-mail: tozzi@arcetri.astro.it \\ 2 Max-Planck Institute for Solar System Research, Max-Planck-Str. 2, 37191 Katlenburg-Lindau, Germany \\ 3 University of Maryland, Department of Astronomy, College Park, MD 20742, USA \\ 4 Institute of Astronomy, Bulgarian Academy of Sciences, Tsarigradsko chaussee 72, 1784 Sofia, Bulgaria \\ 5 European Southern Observatory, Alonso de Cordova 3107, Vitacura, Santiago, Chile \\ 6 Armagh Observatory, College Hill, Armagh BT61 9DG, Northern Ireland \\ 7 Universidad Metropolitana de Ciencias de la Educacion, Av. J.P. Alessandri 774, Nunoa, Santiago, Chile \\ 8 European Southern Observatory, Karl-Schwarzschild-Str. 2, 85748 Garching, Germany \\ 9 Commissariat Energie Atomique, 91191 Gif-sur-Yvette, France \\ ${ }^{10}$ Deutsche Luft- und Raumfahrt Agentur, Rutherfordstr. 2, 12489 Berlin-Adlershof, Germany \\ 11 Vrije Universitet Brussel Pleinlaan 2, 1050 Brussels, Belgium
}

Received 5 April 2007 / Accepted 17 August 2007

\section{ABSTRACT}

Context. On 4 July 2005 at 05:52 UT, the impactor of NASA's Deep Impact (DI) mission crashed into comet 9P/Tempel 1 with a velocity of about $10 \mathrm{~km} \mathrm{~s}^{-1}$. The material ejected by the impact expanded into the normal coma, produced by ordinary cometary activity. The La Silla and Paranal sites of the European Southern Observatory (ESO) in Chile participated in the world-wide campaign to observe this event.

Aims. Based on visible and near-IR observations of the comet, the characteristics and the evolution with time of the cloud of solid particles released by the impact is studied in order to gain insight into the composition of the nucleus of the comet. Also, an analysis of solid particles in the coma not related to the impact was also performed.

Methods. The characteristics of the non-impact coma and cloud produced by the impact were studied by observations in the visible wavelengths, using narrow band filters with passbands free of gas emission, and in the near-IR. The scattering characteristics of the "normal" coma of solid particles were studied by comparing images in various spectral regions, from the UV to the near-IR. For each filter, an image of the "normal" coma was then subtracted from images obtained in the period after the impact, revealing the contribution of the particles released by the impact. Comparison of the images of the cloud recorded in the various filters provides some ideas about the composition of the particles and their evolution.

Results. For the non-impact coma the Af $\rho$, a proxy of the dust production, has been measured in various spectral regions. The presence of sublimating grains, which scatter very efficiently in the near-IR, has been detected. Their lifetime was found to be of the order of $11 \mathrm{~h}$. Regarding the cloud produced by the impact, the total geometric cross section multiplied by the albedo, SA, was measured as a function of the color and time. From the expansion of the cloud, the projected velocity was studied. It appeared to obey a Gaussian distribution with the average velocity of the order of $115 \mathrm{~m} \mathrm{~s}^{-1}$. By comparing the observations taken $3 \mathrm{~h}$ apart, about $20 \mathrm{~h}$ after the impact, we have found a strong decrease in the cross section in $J$ filter, while that in $K_{\mathrm{s}}$ remained almost constant. This is interpreted as the result of sublimation of grains dominated by particles of sizes of the order of some microns.

Key words. comets: general - comets: individual: Comet 9P/Tempel 1 - infrared: solar system

\section{Introduction}

The Deep Impact mission (hereafter DI) to the Jupiter family comet 9P/Tempel 1 (hereafter 9P) was aimed at studying the cratering physics in minor bodies in the solar system and the primordial material preserved inside cometary nuclei. On July 4, 2005 the impactor of the DI experiment produced a high-speed (about $10 \mathrm{~km} \mathrm{~s}^{-1}$ ) impact in the nucleus of 9P excavating a considerable amount of cometary material that was observed and measured both in-situ by the DI fly-by spacecraft and remotely

^ Based on observations performed at the ESO La Silla and Paranal Observatories in Chile (program ID 075.C-0583). by Earth-based instrumentation. First results of the mission are described in A'Hearn et al. (2005) and Sunshine et al. (2006). Early earth-based and other space-based measurements of the event have been published by Meech et al. (2005), Sugita et al. (2005), Harker et al. (2005), Lisse et al. (2006), and Schleicher et al. (2005).

At the European Southern Observatory (ESO) DI received considerable observing time allocated to observe the event at their Chilean observatory, at Cerro La Silla and at Cerro Paranal sites (Kaeufl et al. 2005a). Here, we summarize results from the visible and near-IR measurements of the dust in the cometary coma obtained both shortly before and after the DI event. We 
focus on the dust ejecta properties such as scattering properties, projected velocity, and spatial distribution and their evolution with time. Complementary data from the ESO DI campaign on polarimetric and mid-IR observations as well as on the cometary gas emission and the large-scale coma activity of the comet are described elsewhere (see e.g., Boehnhardt et al. 2007). Pre-impact monitoring of the cometary activity is described by Kaeufl et al. (2005b) and Lara et al. (2006).

\section{Observations}

\subsection{Telescopes, instruments, filters}

The majority of the observations, described here, were performed at the European Southern Observatory (ESO) in La Silla/Chile using the $3.5 \mathrm{~m}$ New Technology Telescope (NTT) by switching between two focal plane instruments: EMMI (ESO Multi-Mode Instrument), for the visible spectral region, and SOFI (Son of ISAAC), for the near-IR ( $J H K)$. Both instruments are of focal reducer type for imaging and spectroscopic observations. EMMI provides a field of view of $9.1 \times 9.9$ arcmin with a two-detector array in the red (400-1000 nm) and of $6.2 \times$ 6.2 arcmin with a single detector in the blue arm (300-500 nm) at 0.32 and $0.37 \mathrm{arcsec} /$ pixel resolution (using the $2 \times 2$ and $1 \times 1$ binning options), respectively. In its large field option used for these observations, SOFI has a single detector of $4.9 \times$ 4.9 arcmin field of view at $0.288 \mathrm{arcsec} / \mathrm{pixel}$ resolution. In the visible, narrow band filters, with bandpasses within selected wavelength regions of interest for cometary science, were used. In particular, for the study of the cometary dust, the following filters with no or negligible gas emission in their passband were used: one in the ultraviolet $\left(U_{\mathrm{c}}\right)$, one in the blue $\left(B_{\mathrm{c}}\right)$ and one in the red $\left(R_{\mathrm{c}}\right)$ spectral region. The near-IR observations were performed with the regular $J, H$, and $K_{\mathrm{s}}$ broad band filters since in this region the gas contamination is negligible. Table 1 gives the log of observations together with the list of filters used including the respective central wavelength and full width at half maximum $(F W H M)$ of the wavelength passband. Technical information on the La Silla telescope and instruments can be found at http://www.1s.eso.org/lasilla/sciops.

Since La Silla was clouded over during night 5-6 July 2006, near-IR imaging of the comet was shifted to the ongoing DI campaign at the Cerro Paranal Observatory using ISAAC (Infrared Spectrometer And Array Camera) at the $8.2 \mathrm{~m}$ unit telescope Antu of the ESO's Very Large Telescope (VLT). Due to the shortage of time at the end of the nightly visibility window, only part of the $J, H$ and $K_{\mathrm{s}}$ filter imaging sequence was performed. ISAAC is a focal reducer-type instrument providing a field of view of $2.5 \times 2.5 \mathrm{arcmin}$ at a pixel resolution of 0.148 arcsec. Technical information on the VLT and the ISAAC instrument can be found at http://wWw . eso.org/paranal/sciops.

\subsection{Calibrations on the sky}

For calibration purposes, some photometric standards were also observed before and after the comet observations on each clear night. In the near-IR, the normal $J H K_{\mathrm{s}}$ photometric standards were used (Persson et al. 1998), while for the calibration of the narrow band filters in the visible, well known spectrophotometric standards from the list by Hamuy et al. (1994) were measured. The required calibration frames (bias and sky flatfield exposures for the visible imaging and screen and/or lamp flatfields with lamp illumination on and off for the near-IR) were obtained during daytime and/or twilight periods.

\subsection{Observing techniques}

The comet imaging was performed with the telescope tracking at the speed of the moving target. Jitter offsets of small amplitude (order of 10-30 arcsec) were applied between individual exposures through a single filter. As usual for extended objects, the observations of the comet in the near-IR spectral region were interlaced by observations of the sky at an offset of $\simeq 8^{\prime}$ in a different region of the sky. A sequence of 5 comet and 5 sky images were usually taken in each near-IR filter. The jitter sequence typically lasted for 11-12 min per filter. Due to the mentioned shortage of time, observations on night July 5-6 with ISAAC consisted of only 2 comet and 2 sky images per filter. Observations in the visible were also repeated 5 times for each filter, offsetting the telescope by 10-30 arcsec. Calibration observations (standards, sky flatfields) were performed with the telescope tracking at the sidereal rate. Daytime calibration images (bias, dome flats) used fixed telescope pointing.

Since EMMI and SOFI focal plane instruments were mounted on the two Nasmyth foci of the NTT telescope, fully simultaneous observations in the near-IR and visible were not possible. However, the switching time between the two instruments was short (less than $15 \mathrm{~min}$ ) and allowed us to use both instruments sequentially during the nightly visibility window of the comet.

The summary log of observations is given in Table 1. During the observing period the Sun $\left(r_{\mathrm{h}}\right)$ and Earth $(\Delta)$ distances of the comet were 1.51 AU and 0.89-0.91 AU, respectively. The phase (Sun-Comet-Observer) angle was $\simeq 41^{\circ}$, and the position angle of the Sun projected on the sky at the position of the comet, was $\simeq 290^{\circ}$.

\section{Data reduction}

\subsection{Frame pre-processing}

For the visible imaging, all comet and standard star images were corrected for the bias and the flatfield. Both bias and flatfield maps were computed as the average of a series of bias and sky flatfield exposures taken during the observing interval and through the corresponding filters (for the flatfield). Subsequently, first-order sky background correction was applied by subtracting the average sky flux value measured at the four edges of the individual flatfielded images.

For the near-IR data the flatfield maps were computed from the screen flat images in each filter with the lamp illumination on and off. Then, for each sequence, a median average sky+bias was computed from the sequence of five sky observations. The comet images were then reduced by subtracting the median averaged sky frame and by dividing the result by the flatfield for the corresponding filter. Finally, comet images for each filter/sequence in the visible and near-IR were obtained as the median average of the single 5 images, after their re-centering on the photometric nucleus. With the median average of 5 images, all the possible background stars and detector defects (hot or dead pixels) were almost completely erased. For the night of July 5-6, this was not possible, since only 2 comet and 2 sky images were recorded. In this case, the background stars and detector defects were erased manually. Although for morphology studies this was acceptable, it prevented any precise quantitative measurements for this particular night. 
Table 1. Filter characteristics and summary log for the comet observation. The table lists on the top the filter name, central wavelength, bandpass $F W H M$ and the instrument, as well as, in the bottom, the starting exposure time for the respective filter imaging performed before (-hours) or after (+ hours) the impact. The sky conditions are indicated in the last line of the table, using the following abbreviations: CLR $=$ clear sky, THN $=$ thin cirrus , COUT $=$ clouded out. On night 5-6/07 the table lists $J H K_{\mathrm{s}}$ imaging from the VLT using ISAAC. $t-t_{0}$ is the time difference between the observation epoch and the DI impact time at the comet. It is given in hours and minutes, with negative values before the impact and positive ones after the event.

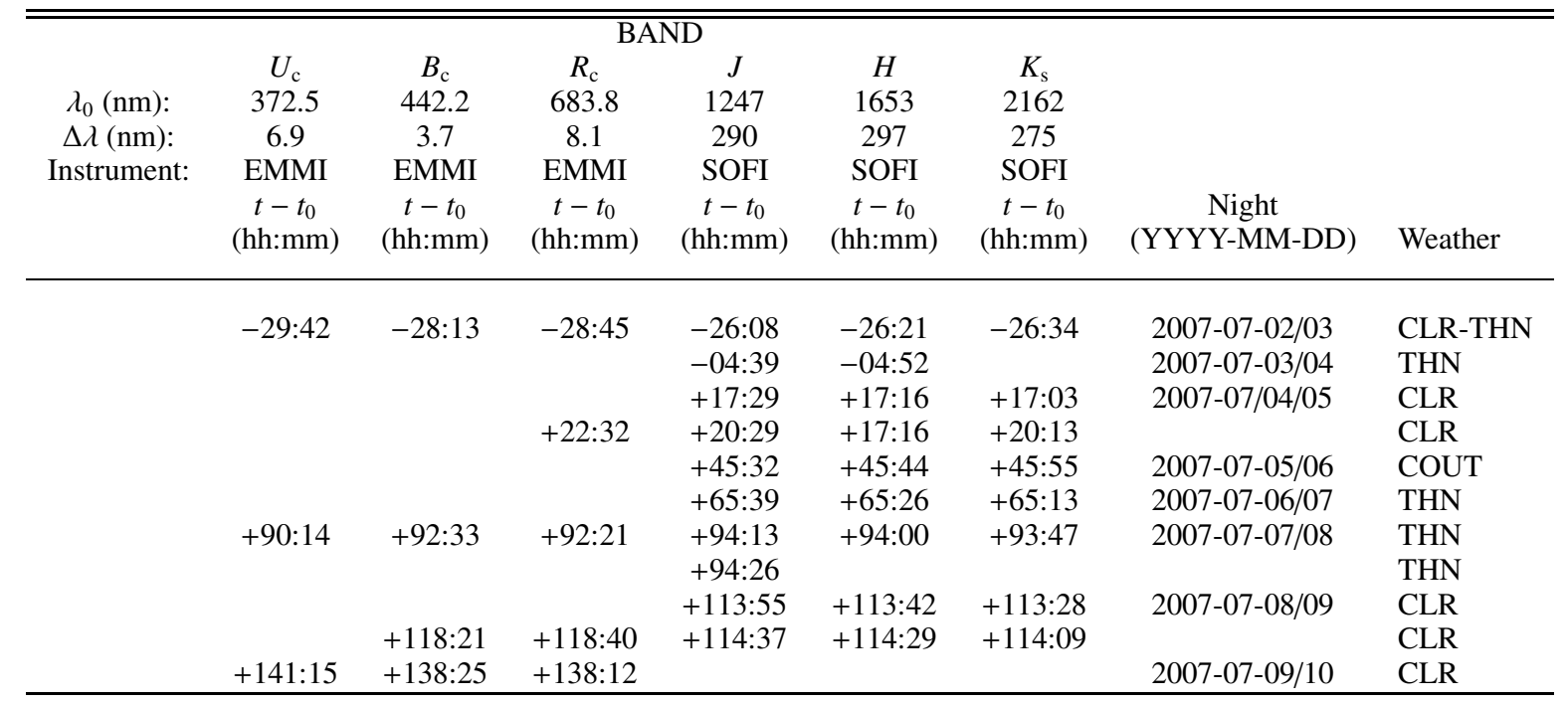

The same procedure was applied to the standards stars. From the reduced standard star images, photometric zero points were derived for clear nights (using aperture photometry and the procedure described in Boehnhardt et al. (2007) for the EMMI images).

\subsection{Residual background flux removal}

The presence of a constant residual background was checked and corrected by measuring the function $\Sigma A f$ at large projected nucleocentric distances, $\rho$. $\Sigma A f$, derived from the $A f \rho$ introduced by A'Hearn et al. (1984), describes the dust albedo $(A)$ multiplied by the total area covered by the solid particles in an annulus of radius $\rho$ and unitary thickness. It is equal to $2 \pi \rho A f$, where $f$ is the average filling factor of the grains at the projected distance $\rho$. Note that the definition given here is slightly different from the the original one given in Tozzi \& Licandro (2002) and Tozzi et al. (2004), even though the physical meaning is the same. Assuming a simple outflow pattern, i.e. geometric attenuation and expansion at constant outflow velocity of the cometary dust, the $\Sigma A f$ function should be independent of $\rho$. In this case, a small residual background (for instance, from incomplete sky subtraction) would introduce a linear dependence of the function with $\rho$. Hence, by applying a trial and error procedure, the residual background can be removed from the reduced images such that the $\Sigma A f$ function becomes constant at large $\rho$. This procedure does not affect the detection of changes in the cometary activity, since the latter introduces an "expanding bump" in the profile (see Sect. 4.2.2), a very different behavior from the linear dependence with $\rho$ introduced by uncorrected background subtraction. This approach for residual background removal is still applicable for the observations taken within about a day after the impact, since the dust produced by the impact was confined to cometary distances shorter than $20000 \mathrm{~km}$ and the coma flux measured in the SOFI images at larger distances could still be used for the above-mentioned calculations. This method of the residual background removal is not easily applicable to the coma images taken with ISAAC during the night July 5-6, 2005 since the DI ejecta had already expanded to the edge of the field of view. For those observations, we assumed that the integral over the position angle (PA) of the function $\Sigma A f$, obtained on the opposite side of the ejecta cloud (over PA between 0 and $90 \mathrm{deg}$ ), remained unchanged from night to night. By changing the background level in ISAAC images, the flux profiles measured in this quadrant before and after DI were forced to be constant with $\rho$. Indications for the assumption of unchanged appearance of the normal coma comes from the analysis of pre-impact observations (see Lara et al. 2006) and from the fact that the coma signal disappears in the respective PA range when subtracting a pre-impact image from a post-impact one (both taken through the same filter).

\subsection{Flux calibration}

All the images taken in clear conditions were then calibrated in Af using the following formula, derived from A'Hearn et al. (1984)

$A f=5.34 \times 10^{11}\left(\frac{r_{\mathrm{h}}}{\mathrm{d} x}\right)^{2} C_{\mathrm{s}} \times 10^{-0.4\left(Z_{\mathrm{p}}-M_{\mathrm{s}}\right)}$

where $r_{\mathrm{h}}$ is the heliocentric distance in $\mathrm{AU}, \mathrm{d} x$ is the detector pixel size in arcsec, $C_{\mathrm{s}}$ is the pixel signal in $\mathrm{e}^{-} / \mathrm{s}, Z_{\mathrm{p}}$ and $M_{\mathrm{s}}$ are the zero points and the solar magnitude in the used filter, respectively. Images taken at non-photometric conditions are flux calibrated assuming that the $\Sigma A f$ profiles at large $\rho$ are coincident with those of the day before and/or day after. This was justified by the fact that, due to the low dust expansion velocity, any change in the dust production would not affect regions at $\rho$ larger than $20000-30000 \mathrm{~km}$ in $24 \mathrm{~h}$. Moreover, the coma analysis by Boehnhardt et al. (2007) suggests that no significant changes in the flux distribution (except for the DI ejecta cloud) took place between July 3 and 10, 2005. The relative calibration from consecutive good nights was checked by comparing the $\Sigma A f$ values of the comet at large nucleocentric distances. 

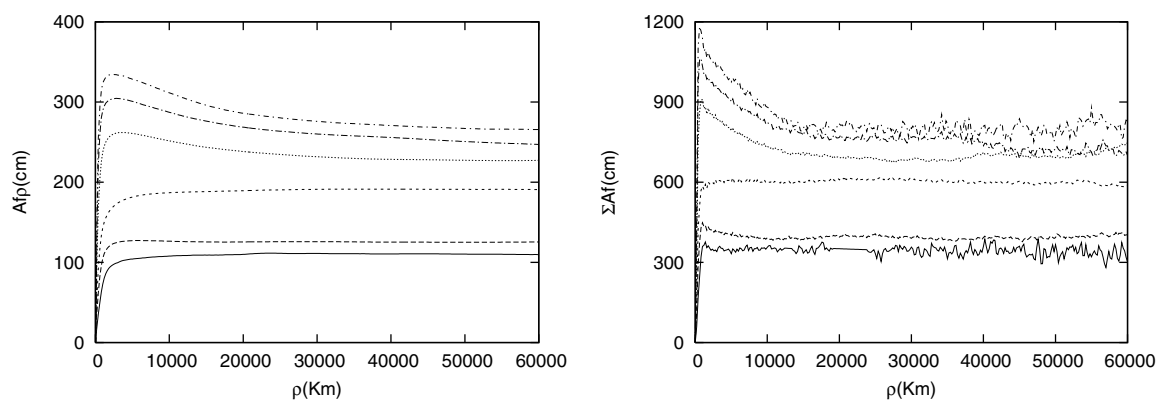

Fig. 1. $A f \rho$ and $\Sigma A f$ profiles determined from the "quiet" comet (see text): $U_{\mathrm{c}}$ (solid line), $B_{\mathrm{c}}$ (long dashed line), $R_{\mathrm{c}}$ (short dashed line), $J$ (dotted line), $H$ (Long dashed - dotted line) and $K_{\mathrm{s}}$ (short dashed - dotted line).

By a careful examination of all comet exposures, we noticed that the images recorded before and $90 \mathrm{~h}$ after the impact were very similar and no or negligible traces of DI ejecta were found. Hence, in order to increase the signal-to-noise (SN) ratio, we computed images (one per filter) of the "undisturbed" comet (hereafter called the "quiet" comet) as the median average of the comet images taken before and after $90 \mathrm{~h}$ from the impact. The standard deviation of the median average is within $2-6 \%$ for the most part of the comet, i.e., for regions at nucleocentric distances between 2000 and $50000 \mathrm{~km}$. In regions closer to the nucleus, this standard deviation increases slightly because of the effect of different seeing in the various nights. It also increases at distances larger than $50000 \mathrm{~km}$ due to the low coma signal.

The subsequent scientific analysis of the calibrated images is based mainly on $\Sigma A f$ profiles and $A f \rho$, both easily obtained by numerical integration of the flux in the comet images in concentric apertures centered on the nucleus. The physical meaning of the $\Sigma A f$ profiles is described above. Following the original definition by A'Hearn et al. (1984), Af $\rho$ is proportional to the average comet flux in the aperture multiplied by its equivalent cometocentric projected distance $\rho$. This function does not depend on $\rho$ for constant outflow velocity. Thus, when using filter images taken in the dust continuum bands, $A f \rho$ is a proxy of the dust production rate, $Q_{\text {dust }}$, of the cometary nucleus. However, due to unknown dust properties such as the dust size distribution and the dust albedo $A$, it is not straightforward to quantify $Q_{\text {dust }}$ using $A f \rho$ measurements of comets.

\section{Results}

\subsection{The "quiet" comet}

\subsubsection{Af $\rho$ and $\Sigma A f$ as a measure of cometary dust production}

The $\Sigma A f$ and $A f \rho$ profiles of the "quiet" comet, derived from the observations of 9P in the six continuum bands $U_{\mathrm{c}}, B_{\mathrm{c}}, R_{\mathrm{c}}, J$, $H, K_{\mathrm{s}}$ during the nights on July $2 / 3,3 / 4,7 / 8,8 / 9$ and $9 / 10,2005$, are plotted in Fig. 1. Various pieces of information on the comet dust production can be derived from these profiles. The horizontal profiles at distances beyond $10000 \mathrm{~km}$ from the nucleus suggest a steady-state level in the dust production that resembles homogeneous and isotropic dust expansion in the coma at a constant speed. From the existence of jet and fan structures in the 9P coma and since the radiation pressure modifies the dust distribution in the coma, it is clear that these ideal conditions are not fulfilled. However, as long as the jets and fans are stable, i.e. they don't change the dust production, the $A f \rho$ and $\Sigma A f$ functions are constant. The solar radiation pressure may introduce a linear dependence of these functions with $\rho$, but it becomes noticeable only at large scales. The $A f \rho$ values as a measure of the dust production of the "quiet" comet are determined at projected
Table 2. Measured $A f \rho$ for the "quiet" comet (see text).

\begin{tabular}{cc}
\hline \hline Filter & $A f \rho(\mathrm{cm})$ \\
\hline$U_{\mathrm{c}}$ & $111 \pm 11$ \\
$B_{\mathrm{c}}$ & $125 \pm 12$ \\
$R_{\mathrm{c}}$ & $191 \pm 19$ \\
$J$ & $228 \pm 23$ \\
$H$ & $253 \pm 25$ \\
$K_{\mathrm{s}}$ & $269 \pm 27$ \\
\hline
\end{tabular}

distances larger than $40000 \mathrm{~km}$. where the radial profiles in Fig. 1 have reached constant values. Results are presented in Table 2.

The error in the Af $\rho$ measurements is mainly due to the relative photometric calibration error, which is estimated to be of the order of $10 \%$. The $A f \rho$ values given here are slightly higher than the value of $112 \mathrm{~cm}$ given by Schleicher et al. (2005) for observations in the green wavelengths $(445-526 \mathrm{~nm})$. They are also higher than the value of $102 \mathrm{~cm}$, later revised to $99 \mathrm{~cm}$, derived from Rosetta/Osiris observations, using the NAC (Near Angle Camera) broad-band filters (Keller et al. 2005; and Keller et al. 2007). However, as already pointed out by Schleicher et al., this may be due to the larger phase angle of the spacecraft observations $\left(69^{\circ}\right)$ compared to the measurements from the Earth $\left(41^{\circ}\right)$.

\subsubsection{Signatures of dust sublimation}

It is evident from Fig. 1 that the near-IR $\Sigma A f$ profiles are not constant. They increase significantly for distances smaller than $\approx 15000 \mathrm{~km}$, showing also a little spike very close to the photometric nucleus. The spike is probably the signature of the nucleus convolved with the seeing. However, the SN ratio of this signature is too low to derive any useful information. Instead, it can be evaluated through the spatial resolution imaging of the coma using adaptive optics systems, such as those collected during the impact week using the NACO instrument at the VLT (not described here). The slow increase of $\Sigma A f$ cannot be due to dynamical phenomena (for example, increased cometary activity), since the near-IR profiles derived from different observing nights look very much the same. Instead the $\Sigma A f$ profiles in the visible do not show any evident increase at small nucleocentric distances.

Similar $\Sigma A f$ profiles have been found in comet $\mathrm{C} / 2000 \mathrm{WM}_{1}$ (LINEAR) (hereafter $\mathrm{WM}_{1}$ ) (Tozzi et al. 2004). At that time this phenomenon was interpreted as a result of sublimation of two kinds of organic grains: one with a lifetime of $\approx 1.3 \mathrm{~h}$ and the other of $\approx 17 \mathrm{~h}$. Following the analysis of the $\mathrm{WM}_{1}$ data, the near-IR $\Sigma A f$ profiles of 9P for $\rho>1000 \mathrm{~km}$ were fit by a function of the kind

$\Sigma A f(\rho)=\Sigma A f_{0}+\Sigma A f_{1} \times \mathrm{e}^{-\left(\frac{\rho}{L_{1}}\right)}$ 
Table 3. $\Sigma A f_{0}$ and $\Sigma A f_{1}$ best fit results (see text).

\begin{tabular}{crr}
\hline \hline Band & $\Sigma A f_{0}(\mathrm{~cm})$ & \multicolumn{1}{c}{$\Sigma A f_{1}(\mathrm{~cm})$} \\
\hline$U_{\mathrm{c}}$ & $345.3 \pm 1.3$ & $10.7 \pm 4.1$ \\
$B_{\mathrm{c}}$ & $377.9 \pm 1.3$ & $62.8 \pm 2.8$ \\
$R_{\mathrm{c}}$ & $603.5 \pm 0.6$ & $2.5 \pm 2.8$ \\
$J$ & $682.0 \pm 0.6$ & $251.0 \pm 1.9$ \\
$H$ & $758.4 \pm 0.9$ & $313.8 \pm 3.1$ \\
$K_{\mathrm{s}}$ & $792.9 \pm 1.6$ & $392.4 \pm 6.3$ \\
\hline
\end{tabular}

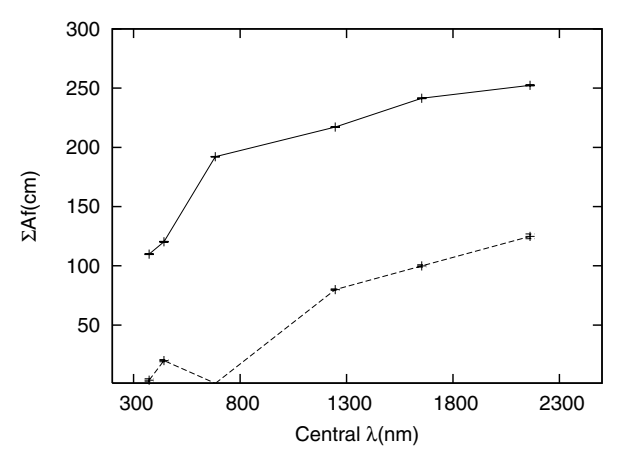

Fig. 2. Wavelength dependence of the permanent (solid line) and sublimating (dashed line) dust components in the coma of comet 9P before the impact. Error bars of the fit parameters $\Sigma A f_{0}$ and $\Sigma A f_{1}$ in Eq. (2) are also plotted, but are too small to be easily seen at the plotted data points.

which contains a constant term $\Sigma A f_{0}$ representing the nonsublimating (permanent) dust component, and just one decaying term $\Sigma A f_{1}$ representing sublimating grains and characterized by the length-scale $L_{1}$. The fit achieved for 9P is very good and shows length-scales $\mathrm{L}_{1}$ of similar value for all three near-IR bands, i.e. $6300 \pm 160 \mathrm{~km}$. Using the fixed length-scale $6300 \mathrm{~km}$, the fitting procedure was done one more time, now for all profiles including those derived from visible data. The best fit parameters $\Sigma A f_{0}$ and $\Sigma A f_{1}$, including standard deviations, are listed in Table 3. Again, the fit gives very good results, with the exceptions of $U_{\mathrm{c}}$ and $R_{\mathrm{c}}$ filters, where $\Sigma A f_{1}$ has values close to zero.

Interesting trends appear when plotting the wavelength dependence of the decaying $\left(\Sigma A f_{1}\right)$ and the constant $\left(\Sigma A f_{0}\right)$ terms of the fits (see Fig. 2). In the near-IR the constant term varies only by a factor of 1.16 going from $J$ to $K_{\mathrm{s}}$, while the decaying term changes by a factor of 1.55 . This finding may indicate that the two solid components are of a very different nature: one (the permanent one) composed of refractory grains and the other (the decaying one) made of sublimating grains (or dust covered by sublimating material) that scatter very efficiently in the nearIR, but are inefficient scatterers in the visible light. As for the comet $\mathrm{WM}_{1}$, taking into account that the length-scale for the density was about $25 \%$ longer than that for the column density, and assuming an outflow velocity of about $0.2 \mathrm{~km} \mathrm{~s}^{-1}$, the lifetime of the sublimating material was of the order of $40000 \mathrm{~s}$ (about $11 \mathrm{~h}$ ). Assuming that the sublimation is driven directly by the radiation, the scalelength and lifetime scale as the square of the heliocentric distance, $r_{\mathrm{h}}$. Then the density length-scale and the lifetime at $r_{\mathrm{h}}=1 \mathrm{AU}$ should be $3500 \mathrm{~km}$ and $17800 \mathrm{~s}$ $(\approx 5 \mathrm{~h})$. That lifetime differs from those found for the volatile dust grains in $\mathrm{WM}_{1}$, which, assuming as well an outflow velocity equal to $0.2 \mathrm{~km} \mathrm{~s}^{-1}$, were $61000 \mathrm{~s}(\approx 17 \mathrm{~h})$ and $4700 \mathrm{~s}(\approx 1.3 \mathrm{~h})$ at the heliocentric distance of that comet (1.2 AU). They scaled to $42000 \mathrm{~s}(\approx 12 \mathrm{~h})$ and $3300 \mathrm{~s}(54 \mathrm{~min})$ at $1 \mathrm{AU}$. The grain sublimation may not depend directly on the solar irradiation, but may

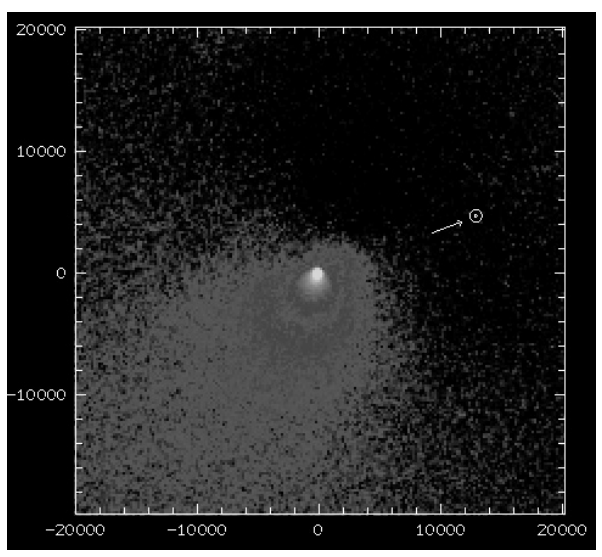

Fig. 3. Difference image of the "quiet" comet minus a synthetic one, as computed from a $K_{\mathrm{s}}$ filter exposure of the comet on July 2-3, 2005 following the procedure described in the text. North is up, East to the left. The Sun direction is indicated. The FoV of the image is $40000 \times 40000 \mathrm{~km}^{2}$ and intensity in $A f\left(0-15 \times 10^{-7}\right)$, with a logarithmic look-up-table. The image shows an enhanced flux level in the southern coma hemisphere due to the sublimating dust and above average activity from isolated regions on the nucleus (jets\&fans). It appears as if the sublimating grains are emitted between PA 100 and $200^{\circ}$.

depend indirectly on it, through the grain temperature. In this case the lifetime and scalelength scale in a more complicated way with $r_{\mathrm{h}}$, dependending on grain size and thermal degradation of the element (see, e.g., Cottin et al. 2004).

The spectral scattering properties of the sublimating grains in $\mathrm{WM}_{1}$ are different from those found here: both $\mathrm{WM}_{1}$ components have a good scattering efficiency in $R$ and $I$, and the long lifetime component has a scattering efficiency similar to the normal dust. The sublimating component in $9 \mathrm{P}$ almost does not scatter in the visible. This means that, even if the phenomena may be similar for the two comets, the nature of the grains must be different.

In order to investigate the origin of the sublimating dust component, we have tried to determine its $2 \mathrm{D}$ distribution in the coma by subtracting a radially symmetric artificial coma image from each near-IR filter image. The artificial coma image was computed using the parameters for the permanent dust component in the respective fit to the original image. Figure 3 shows an example for the difference image in the $K_{\mathrm{s}}$ filter. The nonuniform flux distribution in the images suggests that the sublimating dust is more confined in the coma sector defined approximately by $\mathrm{PA}=100^{\circ}$ to $200^{\circ}$. Given the indicated PA range, the appearance of sublimating dust seems to correlate with the nucleocentric surface regions of enhanced activity since various jet and fan structures are detectable in the same coma region (see Lara et al. 2006; Boehnhardt et al. 2007). However, the jets and fans are also present in the difference images described above since the subtraction of a circular symmetric comet image does not cancel any asymmetries in the coma flux distribution. Thus, it is not possible to fully disentangle the jet and fan structures from the sublimating dust component, except that the former have much lower intensity levels than those of the fading grains.

\subsection{The ejecta cloud}

\subsubsection{Geometry of the ejecta cloud}

For the study of the various effects produced by the DI event, the signal of normal activity was removed from the post-impact 

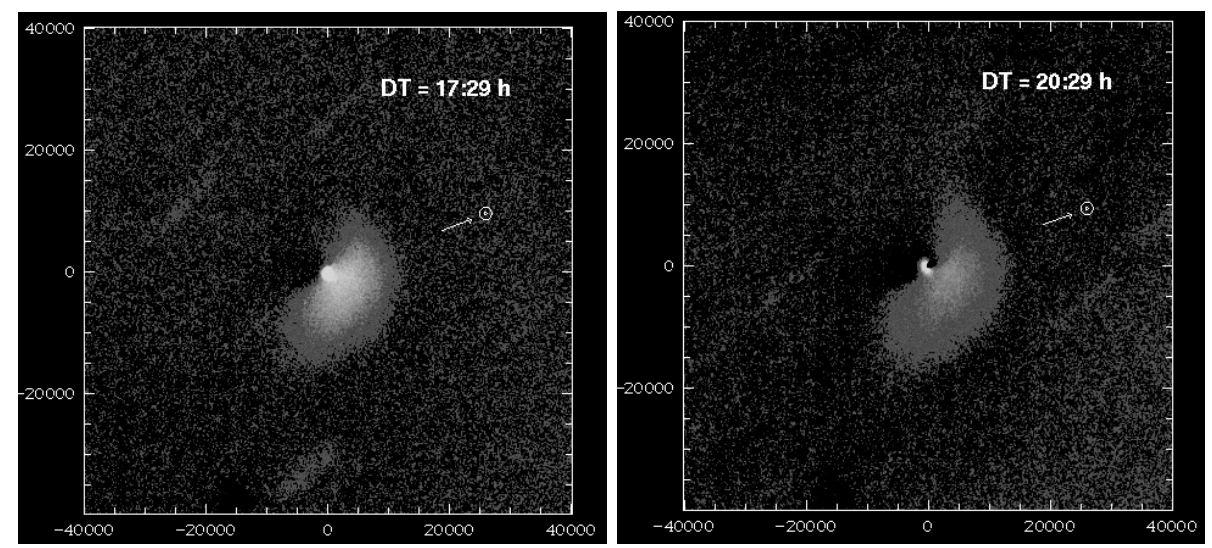

Fig. 4. Processed $J$ filter images of the ejecta cloud recorded about 17 and $20 \mathrm{~h}$. after the impact. The "quiet" comet is removed from the postimpact exposures. The intensity scale is in $A f\left(0-3 \times 10^{-7}\right)$, with a logarithmic look-up-table. North is up, East to the left, the field of view (FOV) at the comet is $80000 \times 80000 \mathrm{~km}^{2}$, the comet nucleus position is in the center of the sub-panels. The Sun direction is indicated by the arrow. The cloud $20 \mathrm{~h}$ after the impact is less bright than $3 \mathrm{~h}$ earlier. The "hole" at the center is an artifact from the subtraction processing of the two exposures pre- and post-impact which had different seeing. Seeing variations do not affect the cloud intensity outside of the typical seeing radius of $1-2$ arcsec, i.e. about $700-1400 \mathrm{~km}$ of the nucleocentric distance.
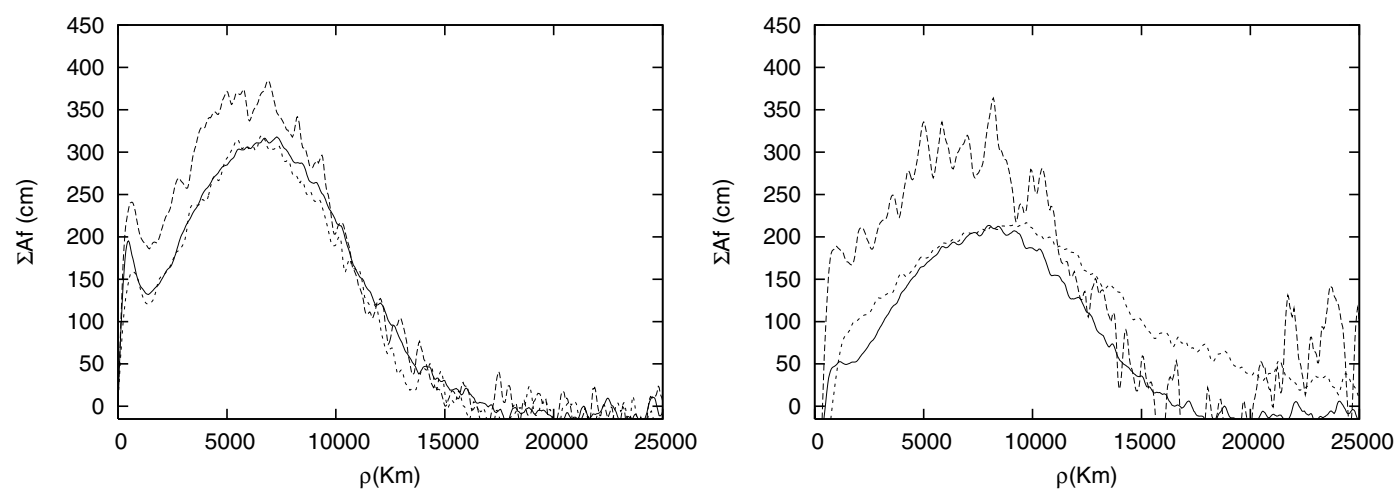

Fig. 5. $\Sigma A f$ profiles of the ejecta cloud vs. $\rho$ measured on night 4-5 July 2005 as a function of $\rho$. Left panel: $J$ (solid line), $H$ (dotted line) and $K_{\mathrm{s}}$ (long dash line) profiles from observations taken 17:29 h after the impact. Right panel: $J$ (solid line), $K_{\mathrm{s}}$ (long dash line) and $R_{\mathrm{c}}$ (dotted line) $\simeq 20: 29 \mathrm{~h}\left(22: 32 \mathrm{~h}\right.$ for $\left.R_{\mathrm{c}}\right)$ post-impact. Note the changes in the $J$ filter profiles between 17:29 and 20:29 h post-impact time.

images of 9P by simple subtraction of the image of the "quiet" comet, taken through the same filter. This processing should remove the non-impact comet coma without introducing new unwanted features from day-to-day variability, since the normal activity of 9P displayed a rather steady-state appearance. The expanding cloud of solid particles is clearly noticeable in the visible and near-IR images until at least July 6-7, 2005 (three days after DI). Figure 4 shows the ejecta cloud in $J$ band as seen 17:29 and 20:29 h after the impact. It can be seen in the figure that the cloud is initially expanding into the coma sector between PA of 120 to $345^{\circ}$. The time evolution of the cloud expansion can be characterized by using visible broadband imaging (Boehnhardt et al. 2007).

\subsubsection{Ejecta dust production}

Integrating the Af in the images difference over the position angle range of the initial ejecta cloud $\left(\mathrm{PA}=120-345^{\circ}\right)$, we obtain the $\Sigma A f$ profile of the cloud vs. $\rho$. The $\Sigma A f$ profiles determined in the $R_{\mathrm{c}}, J, H$ and $K_{\mathrm{s}}$ filters for the night just after the impact are shown in Fig. 5. Note that the different extension of the cloud profiles in the figure is due to the different observing epochs during which the cloud was expanding in the field of view.

By integrating these profiles over $\rho$, we can obtain the total scattering cross section (SA) of the dust ejecta, $\mathrm{SA}=\int(\Sigma A f) \mathrm{d} \rho$, i.e., the albedo at the phase angle of the comet multiplied by the total geometric grain cross section. SA provides useful information to evaluate the number and the intrinsic color of the particles produced by the impact and their evolution with time. For the first observations of the first night (July 4-5, 2005) after the impact, we measured the SA to be $27.6,27.3$ and $34.6 \mathrm{~km}^{2}$ in $J, H$ and $K_{\mathrm{s}}$ respectively. Assuming that the scattering properties of the ejecta grains are the same as those of the refractory component in the pre-impact coma, it is possible to estimate the time interval TI necessary to produce the same amount of dust by the normal activity: $\mathrm{TI}=\frac{\mathrm{SA}}{v \Sigma A f_{0}}$, where $v$ is the mean outflow velocity, and $\Sigma A f_{0}$ are the values obtained for the normal activity. To estimate the order of magnitude, any possible differences in the scattering properties of the dust grains were ignored and a $v=0.2 \mathrm{~km} \mathrm{~s}^{-1}$ was assumed. Then TI is about $5-6 \mathrm{~h}$ (depending on the filter). For the lower velocity $v=0.1 \mathrm{~km} \mathrm{~s}^{-1}$, the equivalent duration of normal dust production is doubled. We conclude that the amount of dust produced by DI and detectable in the near-IR about $17-20 \mathrm{~h}$ post-impact time is equal to the amount of the dust produced during a few hours (maximum half a day) of normal activity of the comet just before the impact.

Results for the effective scattering cross section SA in $\mathrm{km}^{2}$, estimated from the dust filter images available to us, are tabulated in Table 4. The relative error for different filters depends mainly on the respective flux calibration uncertainty that 
Table 4. Effective cross section of the ejecta grains in $\mathrm{km}^{2}$ vs. time $t-t_{0}$ after impact for different dust filters. "Effective cross section" is defined in the text.

\begin{tabular}{ccccc}
\hline \hline$t-t_{0}(\mathrm{hh}: \mathrm{mm})$ & $\mathrm{SA}\left(R_{\mathrm{c}}\right)$ & $\mathrm{SA}(J)$ & $\mathrm{SA}(H)$ & $\mathrm{SA}\left(K_{\mathrm{s}}\right)$ \\
\hline $17: 29$ & & 27.6 & 27.3 & 34.6 \\
$20: 29$ & & 17.9 & & 33.0 \\
$22: 32$ & 27.3 & & & \\
\hline
\end{tabular}

is estimated to be of the order of $10 \%$. The relative error for different measurements with the same filter does not depend on this calibration, because during the data reduction the $\Sigma A f$ profiles of the ejecta cloud images were checked to match the reference profile of the "quiet" comet at nucleus distances beyond $30000 \mathrm{~km}$. Thus, the relative uncertainty in $\Sigma A f$ for different post-impact epochs depends only on the accuracy of the matching of the "quiet" comet profiles. As seen in Figs. 4 and 5, a rather accurate match is achieved and, hence, the relative error of $\Sigma A f$ is evaluated to be less that $5 \%$. The values shown in the table are in agreement with the $33 \pm 3 \mathrm{~km}^{2}$ obtained in the visible range from Rosetta/Osiris observations about $40 \mathrm{~min}$ after the impact (Küppers et al. 2005).

\subsubsection{Ejecta velocities}

Contrary to the natural outbursts in comets, in the case of DI event the exact starting time of the formation of the ejecta cloud is very well known. Hence, assuming that the major dust production by DI was short-term (as suggested by the fly-by spacecraft imaging; see A'Hearn et al. 2005), the radial profiles of the ejecta cloud would also reflect the expansion velocity distribution of the cloud particles. The solar radiation pressure applies some acceleration $\frac{\mathrm{d} v}{\mathrm{~d} t}$ to the particles, which is inversely proportional to the particle radius, $a$. It has been shown that some time between 1 and 2 days after impact the dust grains, as observed in the visible, reached the turning point in their motion in the sunward direction (Boehnhardt et al. 2007) due to the solar radiation pressure.

So, depending on the grain size, the radial profiles $\Sigma A f(\rho)$ of the ejecta cloud show a memory of the initial projected velocities of the dust after the ejection from the nucleus and possible further acceleration in the near-nucleus zone by the ejecta gas and/or normal gas release activity. Thus, $\Sigma A f(\rho)$ divided by the elapse time since the impact results in some distribution function $\Sigma A f(v)$ where $v$ is the mean velocity of the dust grain at the respective projected nucleocentric distance in the cloud. A typical mean velocity distribution is shown in Fig. 6. As pointed out by Küppers et al. (2005) and Jorda et al. (2007) the radial profile of the eject cloud can be well fitted with a Gaussian function. In Fig. 6, the Gaussian function that provides the best fit to the velocity profile is overplotted. The agreement is very good for all the filters. For comparison, a Maxwellian function is also shown. Here the agreement with the data is good in the leading part of the function, but is poor for small velocities.

Because of the radiation pressure (see above), the velocity distribution is not the same in all directions; the mean velocity is lower in the Sun direction than in other directions. To study this effect, the cloud images have been divided in three sectors: S1, S2, and S3 defined by their position angle ranges $145-204^{\circ}$, $205-264^{\circ}$ and $265-325^{\circ}$, respectively. The velocity profiles were then fitted with a Gaussian function. Results are shown in Table 5 , where the mean velocity $\bar{V}$ and its $F W H M$ is given for nights July 4-5 and 5-6. The table does not indicate the errors of the

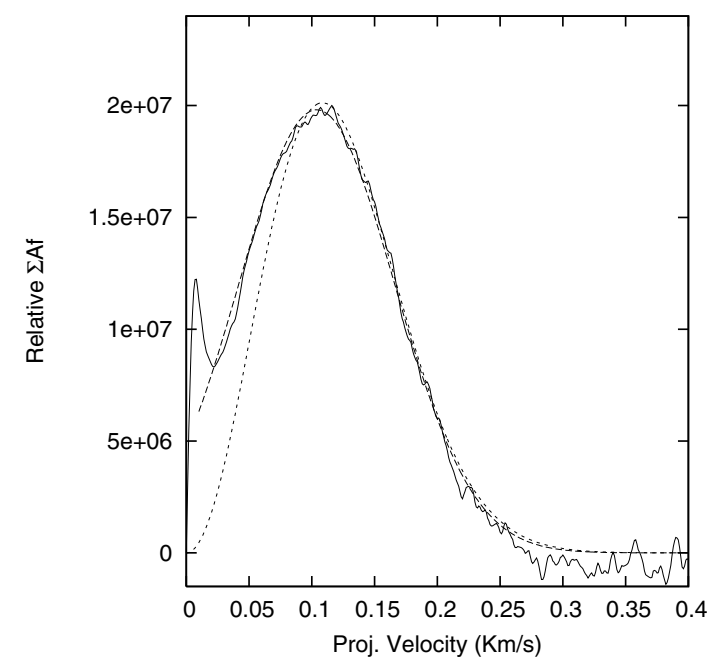

Fig. 6. The $\Sigma A f(v)$ profile, which is proportional to the mean projected velocity distribution of the ejecta dust, as obtained from $J$ filter imaging during night 4-5 July 2005 . The measured profile (solid line) is compared to Gaussian (dashed line) and Maxwellian distributions (dotted lines).

Table 5. Average projected velocities $\bar{V}$ and $F W H M$, in $\mathrm{m} \mathrm{s}^{-1}$, as determined in the three sectors $\mathrm{S} 1, \mathrm{~S} 2$ and $\mathrm{S} 3$ of the dust ejecta cloud (see text) for different filters.

\begin{tabular}{cccccccc}
\hline \hline & $t-t_{0}$ & \multicolumn{2}{c}{ Sector S1 } & \multicolumn{2}{c}{ Sector S2 } & \multicolumn{2}{c}{ Sector S3 } \\
Band & $($ hh:mm) & $\bar{V}$ & $F W H M$ & $\bar{V}$ & $F W H M$ & $\bar{V}$ & $F W H M$ \\
\hline$R_{\mathrm{c}}$ & $23: 32$ & 138 & 95 & 103 & 61 & 87 & 50 \\
$J$ & $17: 29$ & 120 & 69 & 100 & 58 & 98 & 51 \\
$J$ & $20: 29$ & 123 & 57 & 101 & 48 & 100 & 40 \\
$J$ & 45.32 & 134 & 48 & 98 & 44 & 93 & 42 \\
$H$ & $17: 16$ & 117 & 69 & 98 & 56 & 98 & 49 \\
$H$ & $45: 73$ & 133 & 58 & 102 & 53 & 76 & 41 \\
$K_{\mathrm{s}}$ & $17: 03$ & 113 & 71 & 95 & 63 & 91 & 50 \\
$K_{\mathrm{s}}$ & $20: 13$ & 143 & 87 & 84 & 52 & 76 & 41 \\
$K_{\mathrm{s}}$ & $45: 55$ & 122 & 62 & 115 & 54 & 97 & 41 \\
\hline
\end{tabular}

fitting since they are very small, of the order of $\pm 2 \mathrm{~km} \mathrm{~s}^{-1}$ and $\pm 4 \mathrm{~km} \mathrm{~s}^{-1}$ for the first and second night after the impact, respectively.

Table 5 indicates the following: (1) $\bar{V}$, as measured in the near-IR, is independent of the filter, (2) $\bar{V}$ depends on the sector, it is slowest in $\mathrm{S} 3$, but shows very similar values for the three near-IR filters, (3) $\bar{V}$ in sector S3 slightly decreases with time, while in sector $\mathrm{S} 1$ it increases with time.

This picture can be explained by the solar radiation pressure, since the projected Sun direction at $\mathrm{PA} \simeq 290^{\circ}$ falls almost exactly in the middle of sector S3. The average values of the mean velocities, as in the near-IR, are the following: $\bar{V}_{S 1}=123 \pm 12, \bar{V}_{S 2}=96 \pm 7, \bar{V}_{S 3}=93 \pm 10 \mathrm{~m} \mathrm{~s}^{-1}$ for the night July $4-5$ and $\bar{V}_{S 1}=130 \pm 7, \bar{V}_{S 2}=105 \pm 9, \bar{V}_{S 3}=89 \pm 11 \mathrm{~m} \mathrm{~s}^{-1}$ for the subsequent night. Using simple physical considerations, the projected distance covered by a grain in the $S 3$ sector $(\simeq$ Sun direction $)$ is $S_{S 3}(t)=V_{\mathrm{ej}} t-\frac{1}{2} \frac{\mathrm{d} v}{\mathrm{~d} t} \times t^{2}$ and, in the sector $\mathrm{S} 1,\left(\simeq 60^{\circ}\right.$ from the antisun direction) $S_{S 1}(t)=V_{\mathrm{ej}} t+\frac{1}{2} \frac{\mathrm{d} v}{\mathrm{~d} t} \cos (60) \times t^{2}$. With simple algebraic operations the $V_{\text {ej }}$ results are $\left(\bar{V}_{S 3}(t)+\right.$ $\left.2 \bar{V}_{S 1}(t)\right) / 3$. Using the average values given above, the results are $V_{\text {ej }}=113 \pm 16$ and $116 \pm 16 \mathrm{~m} \mathrm{~s}^{-1}$, for the nights July 4-5 and 5-6, respectively. The typical $F W H M$ is of the order of $75 \mathrm{~m} \mathrm{~s}^{-1}$ for both nights. In the visible the eject velocity is estimated to be of the order of $120 \mathrm{~m} \mathrm{~s}^{-1}$. Since only a single observation of this 


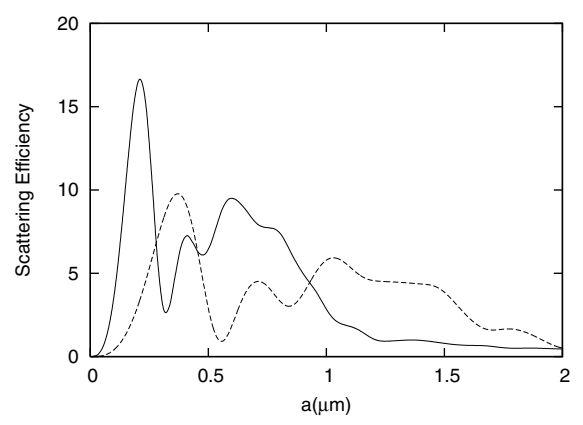

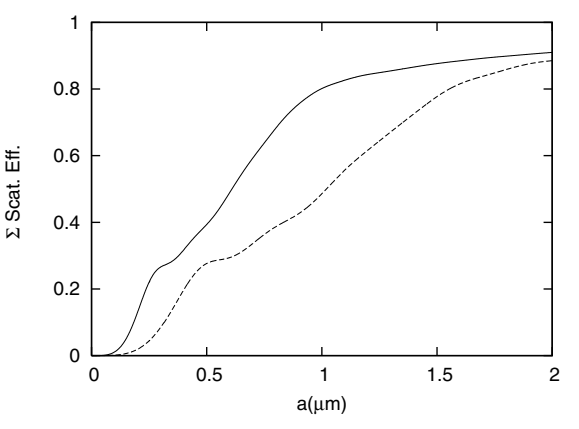

Fig. 7. Differential (left panel) and relative cumulative (right panel) scattering efficiency of a cloud of particles, as a function of their radius (see text), as observed in the filter $J$ (solid line) and in $K_{\mathrm{s}}$ (dotted line). kind is available to us, we adopt an uncertainty similar to those in the near-IR.

\subsubsection{Effective scattering cross section}

A surprising finding is the rapid decrease in the effective $J$ scattering cross section SA of the ejecta cloud by $35 \%$ during the time interval from 17:29 to 20:29 $\mathrm{h}$ after the impact as seen in Table 4. This is also noticeable in Fig. 4, which demonstrates that the cloud at 20:29-h post-impact is significantly fainter than three hours earlier (17:29 h post-impact) even though it has almost the same extension. Also, the change in the radial profiles $\Sigma A f$ of the $J$ filter data 17:29 and 20:29 h after the impact shown in Fig. 5 confirms the change in the scattering of the ejecta particles. This cannot be due to calibration problems, because SA is computed using the difference between the impact images minus the "quiet" comet: calibration problems would give values different from zero in regions far from the cloud. However, this is not the case, as can be seen in Figs. 4 and 5.

The cloud color 17:29 $\mathrm{h}$ after the impact is almost "gray" from $J$ to $H$, but its scattering efficiency increases by $25 \%$ in $K_{\mathrm{s}}$. Three hours later (20:29 h post-impact), the scattering efficiency increases by $84 \%$ between $J$ and $K_{\mathrm{s}}$ (observations in $H$ were not taken because of the lack of time). Thus, the reduction in the effective scattering cross section of the dust cloud in the near-IR between 17:29 and 20:29 $\mathrm{h}$ after the DI event was accompanied by a strong reddening of the dust.

To study this, the scattering efficiency for the three near-IR filters as a function of particle radius, $a$, was modeled. In the model, spherical particles were assumed with a power-law size distribution of power -3.1 as derived by Jorda et al. (2007) using data obtained $40 \mathrm{~min}$ after the impact. The refractive index was set to $1.65+i 0.062$, that represents a mixture of silicates and organics typical for comets (Jessberger et al. 1988). Results are shown in Fig. 7: the left panel gives the scattering efficiency for $J$ and $K_{\mathrm{s}}$ bands as a function of the particle radius, while the right panel gives the cumulative scattering efficiency of the particles, normalized to 1 at large sizes $(5 \mu \mathrm{m})$. From the figures it is possible to see that the particles with radius less that $0.1 \mu \mathrm{m}$ give a negligible contribution to the scattering and that $80 \%$ of the total scattering is reached for the particles with $a \leq 1$ and $\leq 1.5 \mu \mathrm{m}$ for $J$ and $K_{\mathrm{s}}$, respectively. Note that the particles with $a \leq 0.92 \mu \mathrm{m}$ are mainly responsible for the greatest difference between the cumulative scattering in $J$ and $K_{\mathrm{s}}$. Indeed, these particles provide $77 \%$ of the total scattering in $J$ and only $44 \%$ in $K_{\mathrm{s}}$. From these results it is possible to derive an important conclusion: if the destruction or sublimation of particles with $a \leq 0.92 \mu \mathrm{m}$ is responsible for the above-mentioned decrease of SA in the $J$ band, a destruction of $50 \%$ of such particles should decrease the SA by $35 \%$. However, since these particles $(a \leq 0.92 \mu \mathrm{m})$ contribute also to the scattering in $K_{\mathrm{s}}(44 \%)$, this destruction should also produce a $22 \%$ decrease of the SA in $K_{\mathrm{s}}$ filter. Instead, the measured decrease for this filter is only $5 \%$.

A possible conclusion is that the observed particles do not obey a power law size distribution. The results obtained by the DI spacecraft spectrometer indicates that the original DI size distribution was dominated by particles of a few microns in size (A'Hearn et al. 2005; and supporting on-line material of Lisse et al. 2006). The difference between Jorda et al. (2007) and this result may be due to a poor sensitivity of the Jorda et al. measurements to particles of size larger than $1 \mu \mathrm{m}$ as is expected for the measurements in the visual, as pointed out in Jorda et al. Thus, the particles of size $a \approx 1 \mu \mathrm{m}$ could dominate in the ejecta cloud 17:29 $\mathrm{h}$ after the impact and produce a significant contribution to the radiation measured in $J$ filter. It is well known that small grains sublimate faster than large ones because they warm up more efficiently than the larger ones, since the absorption of the solar radiation is proportional to their area and the heating is inverse proportional to their volume. This results in the $1 / a$ law (see, e.g. Lamy 1974), i.e. the smaller the particles are the faster they sublimate. This means that $1 \mu \mathrm{m}$ particles may sublimate faster and be eliminated from the size distribution more efficiently than larger particles. Thus, 20:29 h after the impact the maximum of the size distribution shifts to larger particles. This leaves the $J$ band without the most efficient contributors whereas the situation the $K_{\mathrm{s}}$ band remains almost unchanged. This scenario, consistent with the in situ data, gives a hope that careful simulations of the sublimation of Deep Impact ejecta particles may provide some information about the sublimation rate of the ejecta volatiles, and, thus, may help to identify it.

An alternative scenario would be a change in the particle composition which significantly modified the value of the refractive index. However, this hypothesis cannot explain the observations. First, the most dramatic changes would be expected shortly after the impact when the most volatile components of the dust, e.g. ice, sublimate. For the less volatile components the change should be slow and it is hard to imagine how such a dramatic change happened between 17:29 and 20:29 $\mathrm{h}$ after the impact. Second, we are not aware of any material that may be expected in comets that has a so significant difference in its optical properties between $J$ and $K_{\mathrm{s}}$ filters.

\subsubsection{Color gradient}

Due to the different orientation of the grain velocity vector to the radiation pressure force, radiation pressure has different effects on the dust grains in sectors S1, S2, and S3 of the ejecta cloud. This results in different sorting of dust particles by size for different sectors which may appear as a difference in the dust colors. From the $\Sigma A f(\rho)$ profiles the color distribution of the cloud is computed and the results are averaged over the PA range of the three considered sectors. Since only the near-IR images had 


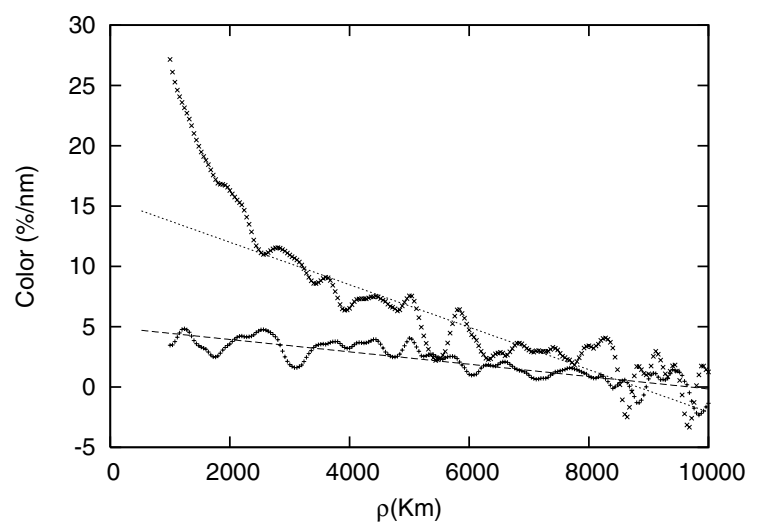

Fig. 8. $K_{\mathrm{s}}-J$ color reddening in $\% \mathrm{~nm}^{-1}$ of the ejecta cloud in sector $\mathrm{S} 3$ as a function of the projected distance from the nucleus. Lower graph is obtained from observations taken 17:29 $\mathrm{h}$ after the impact, the upper graph applies to 20:29 h after DI. Dotted and dashed lines show linear fits to the measurements. While the color remained almost the same at distances $\rho=8000-10000 \mathrm{~km}$ in the cloud, at smaller distances both the color and its spatial gradient increased significantly during the $3 \mathrm{~h}$ between the two observation sequences. Note also the non-linear increase of the reddening function for projected distances below $3000 \mathrm{~km}$, as seen in the data obtained 20:29 $\mathrm{h}$ after impact.

sufficient signal-to-noise ratio within the nucleocentric distance range of $2000-9000 \mathrm{~km}$, and only $J$ and $K_{\mathrm{s}}$ observations were performed during the first night after impact, only the $J-K_{\mathrm{s}}$ color (C) is computed. The results, in percent per nm and per $1000 \mathrm{~km}$, for the night July 4-5, 2005 show that the ejecta cloud becomes "bluer" with larger distance from the nucleus. From the images $\simeq 17: 29 \mathrm{~h}$ after the impact we have found the following: $C_{S 1}=(4.1 \pm 0.1)-(0.31 \pm 0.02) \% \mathrm{~nm}^{-1}(1000 \mathrm{~km})^{-1}$ $C_{S 2}=(3.8 \pm 0.1)-(0.36 \pm 0.02) \% \mathrm{~nm}^{-1}(1000 \mathrm{~km})^{-1}$ $C_{S 3}=(5.0 \pm 0.1)-(0.51 \pm 0.02) \% \mathrm{~nm}^{-1}(1000 \mathrm{~km})^{-1}$.

Thus, the grains closer to the nucleus scattered about $40 \%(4 \%$ $\times$ the difference of the central wavelength of $K_{\mathrm{s}}$ and $J$ ) more efficiently in $K_{\mathrm{s}}$ than in $J$. However, they scatter with almost the same efficiency in sector S3, at about $10000 \mathrm{~km}$ from the nucleus. The strong difference in the gradients between the three sectors can be explained by the effect of the solar radiation pressure mentioned above.

Three hours later (i.e. 20:29 $\mathrm{h}$ after the impact) we find: $C_{S 1}=(7.5 \pm 0.3)-(0.28 \pm 0.06) \% \mathrm{~nm}^{-1}(1000 \mathrm{~km})^{-1}$ $C_{S 2}=(10.7 \pm 0.3)-(1.06 \pm 0.02) \% \mathrm{~nm}^{-1}(1000 \mathrm{~km})^{-1}$ $C_{S 3}=(15.5 \pm 0.3)-(1.76 \pm 0.02) \% \mathrm{~nm}^{-1}(1000 \mathrm{~km})^{-1}$. As is expected from decrease in the effective scattering cross section in the $J$ filter, a significant increase in the dust reddening took place close to the nucleus. The scattering efficiency of the grains close to the nucleus became a factor 2-3 higher in $K_{\mathrm{s}}$ than in $J$. We also notice a strong change in the spatial gradients of the dust reddening: while the gradient in $\mathrm{S} 1$ changes only by about $25 \%$, in the two other sectors the change is significantly more evident, e.g. by factor of 3 for S3. Moreover, the increase is non-linear in the inner part of the cloud, as can be seen in Fig. 8.

These facts cannot be explained by the solar radiation pressure only. Also, from our estimation of the speed of particles, the three hours between our observations cannot bring a significant number of new particles: the particles can travel only $\approx 20 \mathrm{~km}$ farther from the nucleus. Thus, 17:29 h after the impact and $3 \mathrm{~h}$ later we observed particles of the same origin. However, their properties and/or size distribution had changed dramatically. This is confirmed by the above change in the scattering cross sections. The changes in color likely also resulted from the sublimation of particles which had some specific, non-power-law size distribution. This dominance of particles of different sizes is what determines the color and its gradient. The difference in the color gradients may indicate the efficiency of sublimation whose rate increases as particle size decreases (see Sect. 4.2.4).

\section{Discussion}

An important result of these observations has been the discovery of the sublimating component in the coma of the so-called "quiet" comet. This kind of fading grain has been found in comet $\mathrm{C} / 2000 \mathrm{WM}_{1}$, where two components, one with a short and the other with a long lifetime, were found when the comet was at 1.2 AU from the Sun. These components were interpreted as organic grains or refractory grains embedded in organic matter that sublimated while heated by the solar radiation. The sublimating component discovered in comet 9P seems to be different from both sublimating components found in comet $\mathrm{C} / 2000 \mathrm{WM}_{1}$. It scatters very efficiently in the near-IR, but it does not scatter at all in the visible. Scaled to $1 \mathrm{AU}$, its length-scale is of the order of $3500 \mathrm{~km}$ and, assuming an outflow velocity of $0.2 \mathrm{~km} \mathrm{~s}^{-1}$, the lifetime is $18000 \mathrm{~s}(5 \mathrm{~h})$. Note that Cottin et al. (2004) suggested that refractory organic grains, namely polyoxymethylene (POM), may be responsible for the distributed source of formaldehyde, observed in several comets. They computed the scalength and lifetime of the POM grains assuming photolysis by solar radiation and thermal sublimation. With a temperature of grains of $350 \mathrm{~K}$, they computed a POM length-scale of the same order of magnitude as that measured in 9P, depending very little on grain size. It varied from 3300 to $7100 \mathrm{~km}$ for sizes going from 0.1 to $10 \mu \mathrm{m}$.

The following results are obtained for the ejecta cloud produced by the impact:

- the total amount of the dust, multiplied by the albedo, covers a surface of about $30 \mathrm{~km}^{2}$ about $17 \mathrm{~h}$ after the impact, but it drops dramatically for the $J$ band 3 h later;

- the velocity distribution of the solid components had a Gaussian distribution with an average ejected velocity equal to $115 \pm 16 \mathrm{~m} \mathrm{~s}^{-1}$ with a $F W H M$ of the order of $75 \mathrm{~m} \mathrm{~s}^{-1}$;

- the velocities in the projected direction of the Sun are smaller than those in other directions and have similar values for the near-IR bands. Those in the visible have larger values than the near-IR ones;

- from the observations 17:29 $\mathrm{h}$ after the impact, the nearIR color of the grains close to the nucleus was found to be very red and shows a strong gradient with the nucleocentric distance with the highest values in the Sun direction. Three hours later, the near-IR color becomes even more red and the gradient with $\rho$ in the Sun direction increases by a factor $\simeq 3$.

The Gaussian velocity distribution of the particles is puzzling. In the case of gas drag produced by an explosive event that has a timescale shorter than the acceleration time scale, the velocity of a particle with radius $a$ should change as $a^{-1}$. This, combined with a power-law size distribution of power equal to -3.1 (Jorda et al. 2007) would produce a velocity distribution very different from a Gaussian one. If the lifetime of the explosive event was longer than the timescale of the acceleration the ejection velocity distribution with particle sizes would follow a more complex law, but always size dependent (see, e.g. Gombosi et al. 1986). In the case of a natural outburst the velocity distribution 
has been found to follow a Maxwellian one (see, e.g., Schulz et al. 2000). On the other hand, a thermal acceleration of the dust, that would give a Maxwellian distribution, is excluded because it would require a temperature excessively high to accelerate a grain with the mass of the order of $10^{-16} \mathrm{~g}$ at a velocity of a hundred $\mathrm{m} \mathrm{s}^{-1}$. Models of ejecta with grains with a power law size distribution, accelerated by gas drag, give a velocity distribution far from a Gaussian one. Only in the case of grains with almost the same size would the velocity distribution follow that of the grains and it can also became a Gaussian one. The average projected velocity found here is in good agreement with the value of $115 \mathrm{~m} \mathrm{~s}^{-1}$ obtained by by Feldman et al. (2007) from HST observations in the visible just after the impact. It is smaller than those noted by Meech et al. (2005) $\left(200 \pm 20 \mathrm{~m} \mathrm{~s}^{-1}\right)$ and Schleicher et al. (2005) $\left(220 \mathrm{~m} \mathrm{~s}^{-1}\right)$ who refer to the velocity of the leading part of the cloud. For the latter we find 250$300 \mathrm{~m} \mathrm{~s}^{-1}$, in fairly good agreement with their values.

However, our result for the Gaussian velocity distribution of the expanding ejecta cloud differs from that of Jorda et al. (2007) $\left(190 \mathrm{~m} \mathrm{~s}^{-1}\right.$ with $\left.F W H M=150 \mathrm{~m} \mathrm{~s}^{-1}\right)$, derived from Osiris observations on-board Rosetta. This difference cannot be explained with the different viewing geometry of Rosetta spacecraft with respect to an observer on Earth (the difference in aspect angle is just $20^{\circ}$ ). The Osiris and our ESO measurements, obtained at similar wavelengths, are in a good agreement for the total light scattering area of the dust ejecta $\left(33 \mathrm{~km}^{2}\right.$ by Osiris and $27.3 \mathrm{~km}^{2}$ for our $R_{\mathrm{c}}$ observations). An explanation for the different velocity of small and faster grains resulting from dust sublimation a few hours after the impact is thus very unlikely.

The dramatic changes in the total amount of dust observed in $J$ band is another very puzzling result. It is associated with a strong change in the color of the cloud and its gradient with $\rho$. A possible scenario would be a sublimation of the dust grains containing slow-sublimating volatiles such as organics. Water ice is excluded because its sublimation timescale is much shorter than $17 \mathrm{~h}$ (Hanner 1981). If the particles of a few micron size dominated in the original size distribution (as was found from the in-situ data) then sublimation of such particles would result in elimination of the most abundant 1 micron size particles, which are the most efficient in the $J$ band. This will manifest in a significant decrease of the brightness in $J$ band as well as by a change in the dust color.

This means that the scattering area SA of the grains just after the impact must have been much larger than that measured at 17:29 h post-impact, i.e., the quantity of the solid component released by the impact may have been of an order of magnitude larger than the 5-10 h of normal activity derived in Sect. 4.2 without considering sublimation effects. However this is not confirmed by the results of Küppers et al. (2005) who found a light scattering cross section of the cloud in agreement with that found here (see above). It is not possible to obtain more information from the results presented here, but about $17 \mathrm{~h}$ after the impact the size distribution of the grains seems not to follow a power law (if it ever did), but seems "monochromatic".

There is no obvious correlation between the organic grains found in the "quiet" comet and those supposed to be present in the ejecta cloud.

\section{Conclusions}

From observations of gas emission-free regions of the comet 9P/Tempel 1 made before and after the Deep Impact event, the scattering characteristics and the velocity of the ejecta cloud produced by the impact have been measured.

Seventeen and a half hours after the impact, the total area covered by the grains of the ejecta cloud, multiplied by their albedo, was $27-35 \mathrm{~km}^{2}$ in $J H K_{\mathrm{s}}$. Three hours later, it dropped to $\approx 18 \mathrm{~km}^{2}$ in $J$, but remained almost constant in $K_{\mathrm{s}}$. During this interval of time, the $J-K_{\mathrm{s}}$ color gradient with the nucleocentric distance $\rho$ also changed significantly, increasing by a factor of three in the direction of the Sun.

The projected average velocity of the ejected cloud measured in the near-IR was $115 \pm 16 \mathrm{~m} \mathrm{~s}^{-1}$, and was found to be independent of the filter used for the observations and the position angle. Its distribution was very similar to a Gaussian.

It has been shown that all these results cannot be explained assuming that grain size has a power distribution nor it is possible to assume that grains are ejected by gas drag.

While the mechanism of grain ejection is difficult to explain, the behavior afterward can be justified only with the presence of $\approx 1 \mu \mathrm{m}$ size organic grains that are sublimated by the solar radiation.

From the pre-impact and late post-impact observations, the presence of a sublimating component has been detected and interpreted in terms of organic grains that sublimate because of the solar radiation.

\section{References}

A'Hearn, M. F., Schleicher, D. G., Feldman, P. D, Millis R. L., \& Thompson, D. T. 1984, AJ, 89,579

A'Hearn, M. F., Millis, R. L., Schleicher, D. G., et al. 1995, Icarus, 118, 223 A'Hearn, M. F., Belton, M. J. S., Delamere, W. A., et al. 2005, Science, 310, 258 Boehnhardt, H., Pompei, E., Tozzi, G. P., et al. 2007, A\&A, submitted

Cottin, H., Bénilan, Y., Gazeu, M.-C., \& Raulin, F. 2004, Icarus, 167, 397 Feldman, P. D., McCandliss, S. R., Route, M., et al. 2007, Icarus, 187, 113 Gombosi, T. I., Nagy, A. F., \& Cravens, T. E. 1986, Rev. Geophys., 24, 667 Jessberger, E. K., Christoforidis, A., \& Kissel, J. 1988, Nature, 332, 691 Jorda, L., Lamy, P., Faury, G., et al. 2007, Icarus, 187, 208

Hamuy, M., Suntzeff, N. B., Hezthcote, S. R., et al. 1994, PASP, 106, 566 Hanner, M. S. 1981, Icarus, 47, 342

Harker, D. E., Woodward, C. E., \& Wooden, D. H. 2005, Science, 310, 278

Kaeufl, H. U., Ageorges, N., Bagnulo, S., et al. 2005a, Messenger, 121, 11

Kaeufl, H. U., Bonev, T., Boehnhardt, H., et al. 2005b, EMP, 97, 331

Keller, H. U., Jorda, L., Küppers M., et al. 2005, Science, 310, 281

Keller, H. U., Küppers, M., Fornasier, S. Jorda, et al. 2007, Icarus, 187, 87

Küppers, M., Bertini, I., Fornasier, S., et al. 2005, Nature, 437, 987

Lamy, P. 1974, A\&A, 35, 197

Lara, L., Boehnhardt, H., Gredel, R., et al. 2006, A\&A, 445, 1151

Lisse, C. M., VanCleve J., Adams, A. C., et al. 2006, Science 313, 635

Meech, K. J., Ageorges, N., A'Hearn, M. F., et al. 2005, Science, 310, 265

Persson, S. E., Murphy, D. C., Krzeminski, W., Roth, M., \& Rieke, M. J. 1998, AJ, 116, 2475

Schleicher, D. G., Barnes, K. L., Baugh, N. F. 2005, AJ, 131, 1130

Schulz, R., Stüwe, J. A., Tozzi, G. P., \& Owens, A. 2000, A\&A, 361, 359

Sugita, S., Ootsubo, T., Kadono, T., et al. 2005, Science, 310, 274

Sunshine J. M., A'Hearn M. F., Groussin, O., et al. 2006, Science, 311, 1453

Tozzi, G. P., \& Licandro, J. 2002, Icarus, 157, 187

Tozzi, G. P., Lara, L. M., Kolokolova, et al. 2004, A\&A, 424, 325 